\title{
FAITS ET VALEURS EN ESTHÉTIQUE : APPROCHES ET ENJEUX ACTUELS
}

Evangelos Athanassopoulos, Filippo Fimiani, Barbara Formis, Jacinto Lageira

Presses Universitaires de France | « Nouvelle revue d'esthétique »

$2016 / 2 n^{\circ} 18 \mid$ pages 5 à 9

ISSN 1969-2269

ISBN 9782130792512

Article disponible en ligne à l'adresse :

http://www.cairn.info/revue-nouvelle-revue-d-esthetique-2016-2-page-5.htm

\section{Pour citer cet article :}

Evangelos Athanassopoulos et al., "Faits et valeurs en esthétique : approches et enjeux actuels », Nouvelle revue d'esthétique 2016/2 ( $\left.\mathrm{n}^{\circ} 18\right), \mathrm{p} .5-9$.

DOI 10.3917/nre.018.0005

Distribution électronique Cairn.info pour Presses Universitaires de France.

(C) Presses Universitaires de France. Tous droits réservés pour tous pays.

La reproduction ou représentation de cet article, notamment par photocopie, n'est autorisée que dans les limites des conditions générales d'utilisation du site ou, le cas échéant, des conditions générales de la licence souscrite par votre établissement. Toute autre reproduction ou représentation, en tout ou partie, sous quelque forme et de quelque manière que ce soit, est interdite sauf accord préalable et écrit de l'éditeur, en dehors des cas prévus par la législation en vigueur en France. Il est précisé que son stockage dans une base de données est également interdit. 


\section{EVANGELOS ATHANASSOPOULOS, FILIPPO FIMIANI, BARBARA FORMIS ET JACINTO LAGEIRA ${ }^{[*]}$}

\section{Faits et valeurs en esthétique: approches et enjeux actuels}

Sans moraliser l'art et l'esthétique et sans esthétiser les décisions éthiques, nos actions nous conduisent à reconnaître l'entrelacement des faits et des valeurs autant sur le plan pratique que sur le plan langagier, sur le plan de nos comportements que sur nos actes, allant des plus quotidiens aux plus élaborés. Parmi les théories esthétiques récentes qui ont contribué à mettre en évidence les limites des conceptions différenciées entre faits et valeurs, en mobilisant des perspectives théoriques variées - notamment les positions pragmatistes, réalistes ou conséquentialistes -, il apparaît que malgré la divergence de leurs approches et des solutions philosophiques qu'elles préconisent, ces théories s'accordent sur le fait que la séparation radicale est non seulement à rejeter, mais se trouve en contradiction avec nos expériences pratico-morales et pratico-sensibles.

Ainsi, dans un texte célèbre intitulé Fait/valeur : la fin d'un dogme (2002), Hilary Putnam réfute de manière convaincante la dichotomie entre faits et valeurs des plus néfastes pour la réflexion philosophique. En effet, cette dichotomie apparemment indiscutée et indiscutable, ce qui est la nature de tout dogme, laisse en l'état un grand nombre de problématiques, non traitées, non perçues, non examinées. L'analyse de Putnam porte essentiellement sur la théorie et la pratique de la connaissance, mais on peut légitimement l'étendre à d'autres champs, à commencer par celui de l'esthétique, lequel se confronte tôt ou tard à cette question, que l'on défende ou rejette la dichotomie. La maintenir ou la refuser suppose des raisons, mais qui restent le plus souvent implicites, $a$ fortiori en esthétique.

Ce contexte culturel se caractérise par certaines particularités observables aussi dans le champ de l'art, et dans la pratique de la création, où les anciens
*

Evangelos Athanassopoulos (Paris-IPanthéon-Sorbonne) ; Filippo Fimiani (université de Salerno/Paris-I-PanthéonSorbonne) ; Barbara Formis (Paris-IPanthéon-Sorbonne) ; Jacinto Lageira (Paris-I-Panthéon-Sorbonne). 
critères d'évaluation sont désormais devenus caducs. On le constate aisément, poser déjà cette question du maintien ou du rejet de la dichotomie des faits et des valeurs s'inscrit au cœur des problématiques les plus urgentes. Car à bien y réfléchir, la définition de valeur reste opaque : l'œuvre d'art serait-elle jugée selon sa valeur morale, sa valeur marchande, sa valeur plastique? Se ranger pour ou contre la fonction du concept de la valeur en art et en esthétique n'exclut pas un certain nombre de divergences concernant la nature même de ce qu'on entend par valeur.

Si traditionnellement le « fait » était l'œuvre, prise dans toute sa tangibilité, actuellement la matérialité de l'objet ne semble plus faire preuve contre une réfutation. L'art est de plus en plus habité par des œuvres dites "immatérielles », ou « éphémères » et donc peu, ou mal, quantifiables selon les anciens critères d'évaluation esthétique et économique.

Le jugement esthétique, clef de voute de toute pensée philosophique sur l'art, ainsi que l'exercice de la critique semblent donc être mis en péril. Peut-on se passer du jugement ? Dans le cas échéant, l'esthétique serait-elle transformée ? Éradiquée de ses origines mêmes en tant que science du sensible ? Les questions pratico-morales prennent ainsi le dessus sur des questions plus classiquement formelles. La fonction sociale de l'art semblerait donc à privilégier contre une idée radicale de l'autonomie artistique et esthétique. Marcel Mauss, dans son célèbre Essai sur le don, semble ouvrir une nouvelle piste de lecture pour l'évaluation de l'art. L'œuvre serait efficace non pas dans sa forme sensible mais dans ses conséquences tangibles au sein du social.

Les questions sociopolitiques viennent aussi s'intégrer au questionnement esthétique, désormais de plus en plus élargi, où l'autorité des personnes impliquées, leur statut social, leur nationalité, leurs origines, peuvent venir apporter un élément en plus (ou parfois en moins) à la valeur même des œuvres qu'ils/elles créent ou simplement activent par la perception. Car le statut de l'artiste a changé, on pourrait même insinuer qu'il est devenu obsolète. Qui est le producteur de l'œuvre? Si l'œuvre cesse d'être manufacturée et originale, mais devient un artefact industriel, la subjectivité du créateur est mise en cause, et donc aussi la valeur même de l'œuvre produite.

La problématique de la valeur prend ainsi à contre-pied la légitimité d'un supposé " travail » de l'art, au sens où l'invisibilité du processus artistique produit un glissement d'un statut de valeurs d'échange aux valeurs d'usage. Se produit ainsi la célèbre confusion entre l'art et la vie ordinaire. Car c'est dans la vie de tous les jours que les actes et les objets s'utilisent, alors que dans une tractation plus proprement commerciale, voire institutionnelle, ces mêmes actes et objets acquièrent un statut à part entière. 
Faits et valeurs en esthétique : approches et enjeux actuels

De ce glissement fondamental, dont on peut en assigner les prémisses à l'ouvrage fondateur L'Art comme expérience de John Dewey, peuvent témoigner un certain nombre de pratiques artistiques de la néo avant-garde désormais devenues des véritables modalités d'expression en art contemporain. On peut ainsi penser au Nouveau Réalisme (Arman), au Pop Art (Warhol), tout comme aux happenings (Allan Kaprow), à la performance (Yvonne Rainer et la Judson Church) et au mouvement Fluxus (Robert Filliou, Ben Vautier, Nam Jun Paik etc.). Le glissement de l'art comme valeur d'échange à l'art comme valeur d'usage, rompt d'une part avec le monde capitaliste de la marchandise en tant qu'objet, et ouvre un champ de possibles vers une économie plus dilatée, où la fluidité et l'éphémère semblent être devenus les nouvelles valeurs.

Il n'est pas étonnant ainsi que les analyses socio-économiques menées par exemple par Luc Boltanski et Ėve Chiapello (Le Nouvel Esprit du capitalisme, 2001) soient d'un grand intérêt pour les artistes d'aujourd'hui, tout comme des textes de philosophie politique qui analysent la «valeur " actuelle de la démocratie, comme les recherches de Toni Negri ou Paolo Virno, par exemple. L'articulation entre l'économique et le culturel, notamment par l'engagement de plus en plus important des politiques culturelles, permet de comprendre que la valeur culturelle de l'art prédomine parfois sur l'ancienne valeur universelle de celui-ci. La contextualisation de plus en plus pragmatique des œuvres au sein de l'espace social, pourrait leur enlever une qualité universelle et éternelle, tout en maintenant ce que Walter Benjamin appelait l'aura de l'œuvre. Cette « aura " n'est donc pas coupée de son contexte culturel. Les choix et les conséquences des politiques culturelles mettent en avant une nouvelle définition de la valeur esthétique, non plus éternelle et idéale, mais ancrée dans le sensible et dans les enjeux politico-économique d'une situation culturelle spécifique.

\section{CONVERGENCES ENTRE ESTHÉTIQUE ET POLITIQUE : INTERROGER LA VALIDITÉ D'UNE TELLE DISTINCTION}

Les mutations politiques actuelles, tout comme les bouleversements géopolitiques auxquels nous assistons actuellement ne peuvent être ignorés au moment précis où le bassin méditerranéen est de plus en plus agité par des révoltes : Syrie, Tunisie, mais aussi Égypte et Palestine, comment ignorer ces conflits? Comment ne pas poser la question du rôle de l'art dans un tel contexte? Quelle est la valeur de l'art, et quelle serait la force des artistes, là où la famine et la brutalité dominent ? Mais aussi, comment ne pas prendre en compte la profonde crise économique et politique que connaissent les pays fondateurs de ce que nous appelons « Europe », comme la Grèce, l'Italie, l'Espagne et le Portugal ?

Comment définir cette crise à l'aune d'une perspective esthétique ? Que peuvent encore l'art et la réception esthétique dans une période de crise ? Quel est
EVANGELOSATHANASSOPOULOS, FILIPPO FIMIANI, BARBARA FORMIS ET JACINTO LAGEIRA

,


le rôle de l'artiste ? Car le paradoxe consiste aussi à relever que c'est précisément en cette période de crise politique, économique, et même morale, que le grand public semble se tourner le plus vers l'institution muséale. Les musées ne semblent pas souffrir d'une telle crise. Que recherche donc le «peuple» au musée ? Des réponses? Un sens historique et culturel qu' on aurait perdu? Ou simplement un divertissement? Où se situe la valeur éthique, et non pas seulement esthétique, de la rencontre avec l'œuvre d'art?

Le contexte et les mutations actuelles confirment l'idée que la question de la « valeur » et de sa confrontation au concept de «fait» est fondamentale. Car dans un moment historique comme le nôtre, les anciennes hiérarchies ne sont pas seulement critiquées, elles sont sur le point de devenir obsolètes. Quel rôle détiennent les pratiques artistiques? Un rôle d'émancipation, de libération, de fuite, de transformation ? Ou, au contraire, l'art pourrait-il devenir une autre manière d'assujettir les individus à l'institution dominante? Au pouvoir politique du plus fort ? On peut effectivement se demander quel impact social possède encore l'art. Comment la pratique artistique aurait-elle une valeur structurante au sein d'une société fragmentée?

Ainsi, des questions liées aux tensions entre groupes dominants et groupes dominés sont inévitables. On constate que des pratiques ou des croyances qui s'imposent comme des critères d'évaluation contribuent à instaurer des inégalités de statut symbolique entre les groupes sociaux (entre les hommes et les femmes, les blancs et les personnes de couleur, les bien portants et les handicapés, les jeunes et les personnes âgées, les hétérosexuels et les homosexuels, etc.). Dans chacun de ces cas, une différence identitaire est l'objet d'une interprétation culturelle qui tend à conforter la position dominante d'un type jugé normal. Ainsi les questions de hiérarchisation et de différence, de genre, de classe et d'ethnie paraissent également importantes à poser dans ce contexte.

Les questions liées aux problèmes réels de la censure et du droit découlent tout naturellement de cette problématique. Si la figure de l'artiste est centrale, le rôle des institutions politiques et gouvernementales peut devenir étouffant dans des régimes où la liberté d'expression est atteinte. Mais jusqu'où l'expression artistique peut-elle aller? Des questions d'éthiques se posent dans le champ des arts et des biotechnologies. Quelles sont limites? Où situer la limite ? Et pourquoi ? Ces questions se posent de façon différente selon les arts et les pratiques (cinéma, danse, performance, arts plastiques, théâtre, etc.). Une interrogation fine sur le champ disciplinaire des artistes concernés est donc nécessaire, tout autant qu'une distinction culturelle de leur pratique et de leur processus d'inspiration.

Si les débats concernant les faits et les valeurs sont récurrents dans différents champs de la philosophie morale et politique, ou d'autres sciences humaines (notamment en sociologie), débats marqués, par exemple, par la célèbre 
Faits et valeurs en esthétique : approches et enjeux actuels

controverse en 1969 entre Theodor Adorno et Karl Popper sur ces problématiques (De Vienne à Francfort. La querelle allemande des sciences sociales), très peu de choses existent en esthétique et en philosophie de l'art qui traitent frontalement cette dichotomie. Si l'on accepte désormais majoritairement que la fatale dichotomie doive être écartée, combattue et critiquée au sein de l'épistémologie, du droit, de la morale, du sociopolitique, il faut également mettre au jour ces questions dans le champ de l'esthétique au risque de prolonger une réflexion qui ne cadre plus ni théoriquement ni pratiquement avec les évolutions des autres domaines du savoir.

L'esthétique et la philosophie de l'art ne peuvent ignorer leurs fonctions au sein de la culture et des connaissances, leur responsabilité lorsqu'il s' agit de porter son regard sur les contextes sociopolitiques et moraux, bref, leur engagement véritable lorsqu'il faut prendre position sur des débats d'une importance cruciale au regard de leurs retombées concrètes. Cela tient ainsi au renouvellement des outils conceptuels, à l'élargissement des champs d'investigation tout à fait inédits (biotechnologies, questions du genre, écologie, urbanisme et design), lesquels interfèrent directement avec notre existence quotidienne.

Les textes réunis ici sont issus d'un appel à communication effectué dans le cadre du sixième Congrès Méditerranéen d'Esthétique ${ }^{[1]}$ organisé par l'Institut ACTE, UMR 8218, CNRS (université de Paris I-Panthéon-Sorbonne) à la Villa Finaly de Florence, du 24 au 28 juin 2014. L'originalité de cette publication tient au fait que le comité d'organisation du congrès (Evangelos Athanassopoulos, Barbara Formis et Jacinto Lageira) a souhaité que, dans un esprit de collaboration européenne, les textes retenus soient ensuite distribués et proposés à trois revues, italienne (Aisthesis. Pratiche, linguaggi e saperi dell'estetico) et françaises (Nouvelle Revue d'esthétique et Proteus. Cahiers des théories de l'art), en vue d'une publication partagée et simultanée. Après une double évaluation à l'aveugle conforme à sa politique éditoriale, le comité de rédaction de la Nouvelle Revue d'esthétique a sélectionné parmi les propositions en langue française qui lui ont été soumises, les articles qu'on trouvera dans ce dossier.

Cette triple publication n'aurait pas été possible sans le soutien de ces revues et de leurs principaux animateurs : Fabrizio Desideri et Filippo Fimiani pour Aisthesis, Evangelos Athanassopoulos pour Proteus, Carole Talon-Hugon pour la Nouvelle Revue d'esthétique. Nous remercions vivement cette dernière ainsi que l'ensemble du comité de rédaction de la NRE d'avoir accepté l'idée d'une telle collaboration ponctuelle.
EVANGELOSATHANASSOPOULOS, FILIPPO FIMIANI, BARBARA FORMIS ET JACINTO LAGEIRA
1. Les Congrès méditerranéens d'esthétique sont des rendez-vous triennaux inslitués en 2000 par M. le Pr Dionysios Les Zivas, Président de la Société grecque d'esthétique, dans le but de promouvoir la recherche et les échanges scientifiques autour des questions esthétiques dans le bassin méditerranéen. Depuis, le Congrès a acquis une dimension qui dépasse les limites géographiques de son appellation, suscitant l'intérêt de chercheurs venant du monde entier. À ce jour, il a connu six éditions dans différents pays du pourtour méditerranéen : "L'esthétique à l'aube du troisième millénaire " (Athènes, 2000), "Lieux et mémoires " (Tunisie, 2003), "L'imaginaire, le sensible et l'art " (Slovénie, 2006), « Art et temps » (Jordanie, 2008), « Art, émotion et valeur » (Espagne, 2011), et enfin "Faits et valeurs en esthétique : approches et enjeux actuels " (Italie 2014). 